\title{
Sibling rivalry between seeds within a fruit: Some population genetic models*
}

\author{
N. V. JOSHI \\ Centre for Ecological Sciences, Indian Institute of Science, Bangalore 560012, India \\ MS received 30 November
}

\begin{abstract}
Competition between seeds within a fruit for parental resources is described using one-locus-two-allele models. While a "normal" allele leads to an equitable distribution of resources between seeds (a situation which also corresponds to the parental optimum), the "selfish" allele is assumed to cause the seed carrying it to usurp a higher proportion of the resources. The outcome of competition between "selfish" alleles is also assumed to lead to an asymmetric distribution of resources, the "winner" being chosen randomly. Conditions for the spread of an initially rare selfish allele and the optimal resource allocation corresponding to the evolutiona rily stable strategy, derived for species with $n$-seeded fruits, are in accordance with expectations based on Hamilton's inclusive fitness criteria. Competition between seeds is seen to be most intense when there are only two seeds, and decreases with increasing number of seeds, suggesting that two-seeded fruits would be rarer than one-seeded or many-seeded ones. Available data from a large number of plant species are consistent with this prediction of the model. •
\end{abstract}

Keywords. Intrabrood conflict; sibling rivalry; brood reduction; cainism; seed-seed competition; evolutionarily stable strategy.

\section{Introduction}

Like many of Haldane's other contributions to population genetics, his model of family level selection (Haldane 1924) also has a much broader range of applications. By comparing the rate of increase of the frequency of the allele favoured by natural selection when competition between individuals was population-wide to the rate when competition was restricted to members of the same family, Haldane demonstrated that the process of family level selection was relatively slower, almost by a factor of two (Haldane 1924; Sutter 1968). However, the formalism developed by him is ideally suited for modelling evolutionary processes that in fact operate at the level of the family, e.g. parent-offspring interactions and sibling rivalry. In this paper, the evolution of sibling rivalry in plants is investigated using methods similar to Haldane's family-level (familial) selection models.

In many bird species, there is an intense and often violent competition between chicks in the same clutch for the food brought by the parents. Indeed, earlier models that described sibling rivalry were inspired by bird species (O'Connor 1978; Stinson 1979), though more general models had also been formulated (Macnair and Parker 1979). It was quickly realized, however, that competition among seeds in a fruit is an equally valid example of intrabrood conflict, is equally intense, and can be described by similar models (Nakamura 1980; Kress 1981). Inclusive fitness models (Westoby

\footnotetext{
* Based on a talk given at the Haldane Centenary Symposium held on 6 November 1992 at Ahmedabad as part of the 58th Annual Meeting of the Indian Academy of Sciences.
} 
and Rice 1982; Queller 1983) as well as genetic models (Law and Cannings 1984) have been used to examine the various details of this phenomenon. Uma Shaanker et al. (1988), in addition to presenting a broad review of brood size patterns in plants, have developed models describing parent-offspring as well as offspring-offspring conflict. Lovett Doust and Lovett Doust (1988) view these processes from an even broader view, that of plant reproductive strategies.

While the present investigation draws on the earlier studies, it differs from them in simultaneously considering (a) an explicit population genetic model for $n$ =seeded fruits, using the fitness function commonly used for plant systems; (b) an asymmetric outcome of competition between identical genotypes; (c) separate and explicit conditions for the spread of the selfish allele and for the evolutionarily stable strategy (ESS); and (d) the relative rarity of two-ovuled plant species, and offering a possible explanation for it.

\section{The model}

An infinite, panmictic population of a purely cross-pollinating species with nonoverlapping generations is considered, with selection operating on two alleles-the recessive normal $(\mathrm{N})$ and the dominant selfish $(\mathrm{S})$. The situation where there are two seeds per fruit is described in detail here, while generalization to $n$-seeded fruits is developed in the appendix.

\subsection{Competition between seeds}

Since there are two seeds per fruit, and there are two phenotypes of seeds (normal and selfish), there will be three types of fruits:

(i) In a fruit with both seeds of the normal type, resource is assumed to be shared equally, and the fitness of each seed is assumed to be unity.

(ii) In a fruit with one selfish seed and one normal seed, the selfish one acquires a higher share of resources from the maternal parent, and thereby acquires a fitness of $1+b$ (where $b$, the benefit, $>0$ ). The normal seed, which consequently gets a reduced level of resource, obtains a fitness of $1-c$ (where $c$, the cost, $>0$ ).

(iii) In a fruit where both the seeds are selfish, either of them is likely (with equal probability) to acquire a larger share of resource. This could be, for example, because one of the ovules is fertilized earlier than the other, or is closer to the resources (see Lee (1988) for a review). Each one thus obtains fitness $1+b$ or $1-c$ with a probability of half.

\subsection{Spread of the selfish allele $S$}

Let $y(\simeq 0)$ be the frequency of NS heterozygotes (and $1-y$, the frequency of NN homozygotes). This implies the frequency of pollen of haplotype $S$ to be $y / 2$.

The NS heterozygote maternal parents would develop three types of two-ovuled flowers, $(N, N),(N, S)$ and $(S, S)$ (where $N$ and $S$ denote the haploid state of the egg within the ovule) in the proportion $0 \cdot 25: 0 \cdot 5: 0 \cdot 25$. The frequency of flowers containing an S-type ovule, and the frequency of the S-type pollen, are both very small (of order 
$y$ ); and in the first-order approximation, terms of order $y^{2}$ can be neglected. This means that flowers containing S-type ovules (one or two) will be fertilized only by $\mathrm{N}$-type pollens, while the (N,N) flowers will be fertilized by at most one S-type pollen, the other one always being type N. In other words, frequencies of (NS, NS), (NS, SS) and (SS, SS) fruits obtained by pollination by two S-type pollens will be vanishingly small and hence can be neglected in the first-order approximation.

Thus, the frequency of (NN, NS) fruits

$$
\begin{aligned}
& =y(1-y) / 2 \quad(\text { from }(\mathrm{N}, \mathrm{S}) \text { type of flowers fertilized by N-type pollen }) \\
& +(y / 2) \cdot 2(1-y) \quad \begin{array}{l}
\text { from }(\mathrm{N}, \mathrm{N}) \text { flowers fertilized by one } \mathrm{N} \text { and one } \mathrm{S} \\
\text { pollen })
\end{array} \\
& =1.5 y,
\end{aligned}
$$

and the fitness of the (NS) seed is $1+b$.

On the other hand, the frequency of the (NS, NS) fruits

$$
=y(1-y) / 4 \quad \text { (from the }(\mathrm{S}, \mathrm{S}) \text { types of flowers fertilized by N-type pollen) }
$$

and the fitness of each NS seed is $1+(b-c) / 2$, and the net contribution of the two seeds is $2+b-c$. Hence the frequency of NS in the next generation, $y^{\prime}$, is given by

$$
\begin{aligned}
y^{\prime} & =[1 \cdot 5 y(1+b)+0 \cdot 25 y(2+b-c)] / 2 \\
& =y(1+(7 b-c) / 8) .
\end{aligned}
$$

The allele $\mathrm{S}$ will increase in population if $y^{\prime}>y$, i.e. if $(7 b-c) / 8$ is a positive quantity, i.e. if $7 b>c$.

The condition for the spread of the selfish allele thus turns out to be

$$
b>c / 7
$$

where $b$ and $c$ denote benefit to the usurper and cost to the other seed, as described earlier.

\subsection{Evolutionarily stable strategy}

Consider now competition between two selfish alleles, the recessive $\mathrm{S}$ which leads to benefit $b$ and cost $c$ and the dominant $S^{\prime}$ which leads to benefit $b^{\prime}$ and cost $c^{\prime}$.

Clearly, the fitness of an SS seed from an (SS, SS) fruit would be $1+(b-c) / 2$ as described earlier, while that of an $\mathrm{SS}^{\prime}$ seed from an $\left(\mathrm{SS}^{\prime}, \mathrm{SS}^{\prime}\right)$ fruit would be $1+\left(b^{\prime}-c^{\prime}\right) / 2$. For an (SS, $\left.\mathrm{SS}^{\prime}\right)$ fruit, the probability of $\mathrm{SS}^{\prime}$ being the winner is $0 \cdot 5$, which gives it a fitness of $1+b^{\prime}$. On the other hand, the probability of SS being the winner is also $0 \cdot 5$, whence the loser $\mathrm{SS}^{\prime}$ obtains a fitness of $1-c$. The fitness of the $\mathrm{SS}^{\prime}$ seed in an $\left(\mathrm{SS}, \mathrm{SS}^{\prime}\right)$ fruit is thus $1+\left(b^{\prime}-c\right) / 2$.

As before, assuming $z$ to be the frequency of $S^{\prime}$ heterozygotes, $1-z$ the frequency of SS homozygotes, and $z / 2$ the frequency of $\mathrm{S}^{\prime}$ pollen, the average fitness of $\mathrm{SS}^{\prime}$ is given by

$$
\begin{aligned}
& {\left[1 \cdot 5 z\left(1+\left(b^{\prime}-c\right) / 2\right)+0 \cdot 25 z\left(2+b^{\prime}-c^{\prime}\right)\right] / z} \\
& \quad=2+b^{\prime}-3 c / 4-c^{\prime} / 4
\end{aligned}
$$

On the other hand, fitness of SS is $2+b-c$. For $S^{\prime}$ to be able to invade the $S$ 
population

$$
\begin{aligned}
& 2+b^{\prime}-3 c / 4-c^{\prime} / 4>2+b-c, \\
& \text { i.e. } b^{\prime}-c^{\prime} / 4>b-c / 4 .
\end{aligned}
$$

In other words, a strategy corresponding to the resource allocation that maximizes $b-c / 4$ will be able to invade any other strategy corresponding to a different allocation and in turn will be uninvadable by any other strategy. Thus the allocation which maximizes $b-c / 4$ is the evolutionarily stable strategy (ESS).

The pollen that fertilize the two ovules in the flowers are assumed to come from different plants. The two seeds in the fruit are, therefore, half-sibs (same mother, different father), and the coefficient of relatedness between the two seeds is therefore $0 \cdot 25$. The quantity $b-c / 4$ thus turns out to be the change in the inclusive fitness (Hamilton 1964) of the dominant seed. The ESS is thus the one that maximizes the change in the inclusive fitness, which in the present situation is the same as maximizing inclusive fitness itself.

\subsection{Resource-fitness relationship}

For a seed to be viable, a minimum threshold level of resource must be invested in it. Thereafter, the increase in fitness (or survival) of the seed with increasing investment of resource obeys the law of diminishing marginal returns. The functional form

where

$$
\text { fitness } \propto\left(R-R_{\min }\right)^{x},
$$

$$
0<x<1 \text {, }
$$

is normally used to describe such a relationship in plants (Smith and Fretwell 1974). In this expression, the power $x$ indicates the efficiency of the seed in converting resources into fitness; thus a higher value of $x$ corresponds to a higher level of efficiency. Without loss of generality, $R_{\min }$ can be assumed to be unity. The optimal parental investment per seed can then be shown to be equal to $1 /(1-x)$ (see, for example, Ganeshaiah et al. 1991).

In the present model, it is assumed that the parental allocation of resources to a fruit is in accordance with the optimal investment per seed. Thus, a two-seeded fruit obtains an investment of $2 /(1-x)$. This is turn in split between the two seeds according to their competitive ability. It is further assumed that the value of $x$ is the same for all the genotypes, i.e. the efficiency of the selfish seed in converting the higher share of resources obtained by it into fitness is assumed to be the same as that of a normal seed.

When the investments are equal, each seed obtains a fitness

$$
F=\left(\frac{1}{1-x}-1\right)^{x}
$$

Let $f$ be the fraction of the total resource obtained by the selfish phenotype $(f>1 / 2)$. Its fitness is then given by

$$
F^{\prime}=\left(\frac{2 f}{1-x}-1\right)^{x} \text {. }
$$


The remaining resource is invested in the other seed, and its fitness is given by

$$
F^{\prime \prime}=\left(\frac{2(1-f)}{1-x}-1\right)^{x}
$$

Since the fitness of the selfish seed is $1+b$ and that of the seed with lower resource is $1-c$, relative to the fitness of the normal seed in a $(\mathrm{NN}, \mathrm{NN})$ fruit, the expressions for benefit and cost turn out to be

and

$$
b=F^{\prime} / F-1
$$

$$
c=1-F^{\prime \prime} / F
$$

It should be borne in mind that, should the level of investment in a seed fall below unity, it does not survive, and the cost becomes equal to 1, regardless of the actual level of investment.

Using equations (1), (2) and (3), for a given value of $x$ it is possible to compute the values of $b$ and $c$ for any level of usurpation $f$ shown by the selfish allele. Since $f$ can vary between $1 / 2$ (equal investment) and 1 (all the resource garnered by the selfish allele), it is possible to obtain the range of $f$ values (for a given value of $x$ ) where $b>c / 7$, i.e. where the selfish allele can invade the population of normal allele.

Similarly, it is possible to obtain the ESS in terms of $f$, for a given value of $x$. It is seen that maximization of $b-c / 4$ is equivalent to

the maximization of $\frac{F^{\prime}}{F}-1-\left(1-F^{\prime \prime} / F\right) / 4$,

i.e. maximization of $\left(F^{\prime}+F^{\prime \prime} / 4\right) / F$,

i.e. maximization of $4 F^{\prime}+F^{\prime \prime}$ with respect to $f$.

It is seen that the value of $f$ corresponding to this optimum, $\hat{f}$, is given by

$$
\hat{f}=\frac{1}{2}+\frac{1}{2}\left[\frac{x\left(1-4^{1 /(x-1)}\right)}{1+4^{1 /(x-1)}}\right] \text {. }
$$

Let $\hat{I}$ be the value of $b-c / 4$ obtained corresponding to $\hat{f}$. When all the resource is garnered by the selfish seed, i.e. when $f=1$, then $c=1$, and let $I^{*}$ be the corresponding value of $b-c / 4$. Using this notation, if $I^{*}>\hat{I}$, then $f=1$ will be the ESS.

\section{Results and discussion}

\subsection{Spread of the selfish allele}

The population genetic model described above for two-seeded fruits indicates that a selfish allele would spread in the population if $b>c / 7$. Since the coefficient of relatedness between two seeds is 0.25 (section 2.3), it might seem that Hamilton's (1964) inequality would suggest the condition to be $b>c / 4$. However, the outcomes of competition between related and unrelated seeds are different; a selfish seed is assumed to always outcompete a normal (not carrying the $S$ allele, hence unrelated) seed, thereby obtaining a fitness $b$. It is only when the competiton is between two selfish (carrying $\mathrm{S}$, hence related) seeds that a non-zero cost is encountered. When 
the probabilities of occurrence of these two situations are taken into account (as in the derivation), the condition $b>c / 7$ is obtained. The tolerance of the higher cost is thus due to a higher probability of benefit being accrued from competition against a normal (hence unrelated) seed.

As seen from the appendix (A1), the condition for the spread of the selfish allele in species containing $n$ seeds per fruit is given by

$$
b>c\left[\frac{(n-2) 2^{n-1}+1}{(n+2) 2^{n-1}-1}\right] .
$$

Thus, for example, for three-seeded and four-seeded fruits, the conditions for the spread of the selfish allele are $b>5 c / 19$ and $b>17 c / 47$ respectively. The spread of selfishness thus seems to become less likely as the brood size increases (figure 1), as expected.

The conditions obtained above can be used to investigate the evolution of brood reduction. If the entire resource is acquired by the dominant seed (or if the quantity of resource available for the other seeds is less than one unit per seed), only the dominant seed survives, and the others are aborted. When one examines the dynamics of the spread of such an excessively selfish allele (the brood reducer, i.e. the one which usurps the entire resource) for different values of $x$, the exponent of the fitness function, it is seen that for values of $x<0.041, b$ cannot be more than $c / 7$. This is intuitively reasonable, as low values of $x$ correspond to low efficiency of conversion of resources into fitness. For lower values of $x$, the increase in fitness obtained by acquiring additional resource is not commensurate with the loss in fitness due to the loss of siblings.

The critical values of $x$ (i.e. those values below which the brood reducer allele cannot successfully invade a population of normal alleles) for three-seeded and four-seeded species (obtained using the methods described in section 2.4) are 0.068 and 0.086 respectively, and increase with $n$, the number of seeds in a fruit (figure 2), for low values of $n$, further highlighting the constraints imposed on the extent of sibling rivalry by higher seed numbers.

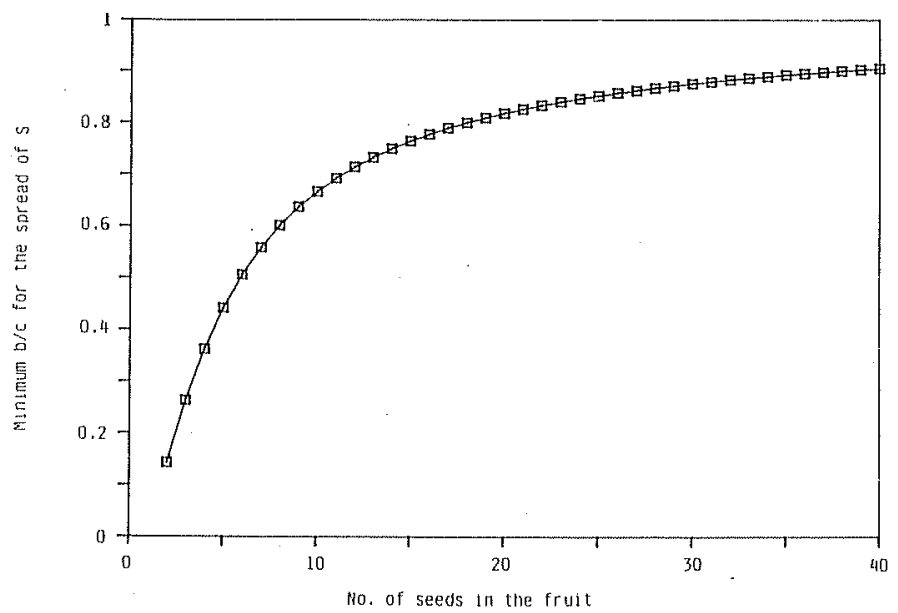

Figure 1. The threshold of $b / c$ that has to be exceeded for a selfish allele to spread in the population as a function of $n$, the number of seeds in a fruit. 


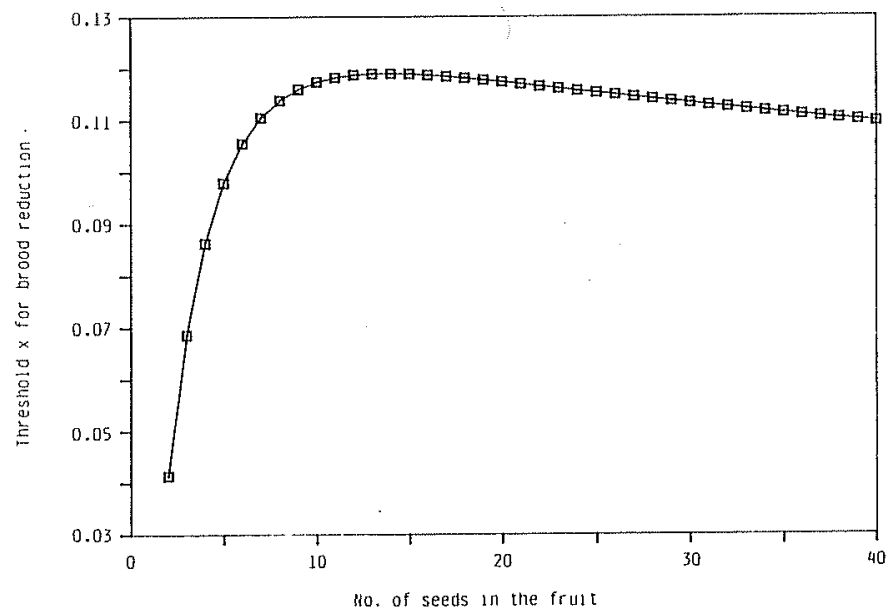

Figure 2. The critical value of $x$ (the exponent in the fitness function, indicative of the efficiency of conversion of resource into fitness), below which a brood reducing allele (i.e. the one that acquires the entire resource for itself, thereby leading to the death or abortion of the rest of the seeds in the fruit) cannot spread in a population (of normal, $\mathrm{N}$-type alleles), as a function of $n$, the number of seeds in a fruit.

For higher values of $n$ (beginning with $n=15$ ), the critical value of $x$ starts decreasing with increasing $n$ (figure 2), though rather slowly. The reason for this lies in the functional dependence of $b, c$, and the threshold of $b / c$ necessary for the spread of the selfish allele on $n$, the number of seeds in a fruit. The benefit $b$ increases slowly, but steadily, with $n$. The cost $c$ is always equal to unity, since seeds other than the dominant one do not survive. The threshold increases rapidly with $n$ initially, but is constrained to reach a value of unity asymptotically. As a result, for low values of $n$, the rate of increase of the realized value of $b / c$ is lower compared to the rate of increase of the threshold with increasing $n$. Consequently, higher values of $x$ are needed for brood reduction to evolve as $n$ increases in the initial stages ( $n=2$ to 14). Beyond this range, however, while the benefit continues to increase, the increase in the threshold is negligible. Hence, smaller values of $x$ are adequate to enable $b / c$ to exceed the threshold. The critical value of $x$ necessary for the brood reducing allele to spread thus starts decreasing with increasing $n$. However, one of the assumptions of the model, viz. that the rest of $n-1$ seeds (i.e. those other than the dominant one) obtain an identical share of the remaining resource, would be quite unrealistic in such a situation. The consequences of using different, more appropriate forms of resource allocation are being investigated.

\subsection{Evolutionarily stable strategy}

For two-seeded species, the optimal fraction $\hat{f}$ of the resource, acquired by the seed with the selfish phenotype (i.e. the value which makes the corresponding allele uninvadable), is equal to $0 \cdot 5$ for $x \simeq 0$ and increases with $x$, reaching $\hat{f}=1$ as $x$ reaches unity. However, this optimum (in the sense of maximization of $b-c / 4$ ) is only a local one. For $x>0 \cdot 1017$, it turns out that $\hat{f}=1$ is the ESS. 


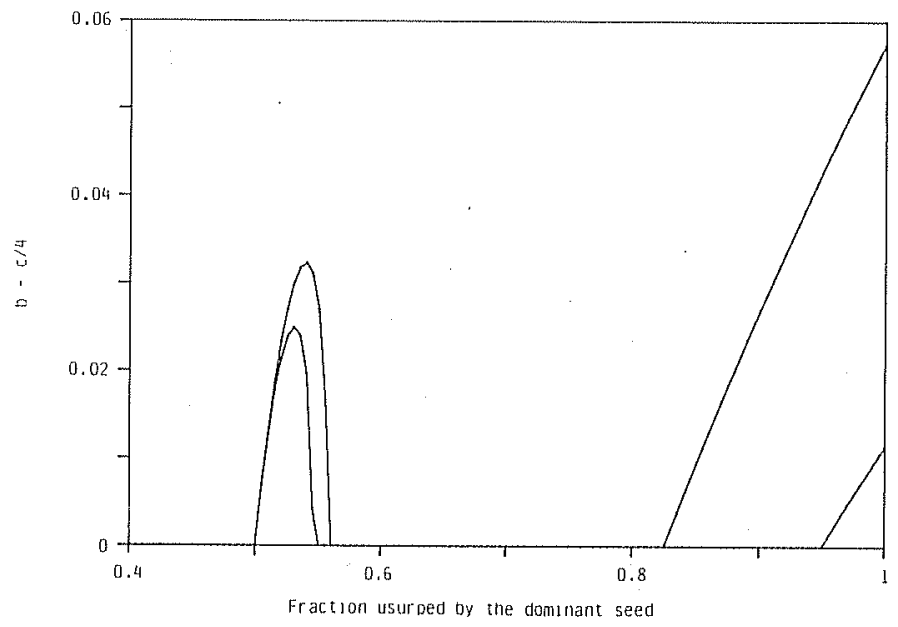

Figure 3. The value of $b-c / 4$ as a function of $f$, the fraction of the resource usurped by the dominant seed, for two different values of $x$. The lower (and inner) curve corresponds to $x=0.095$, while the upper (and outer) curve corresponds to $x=0.12$.

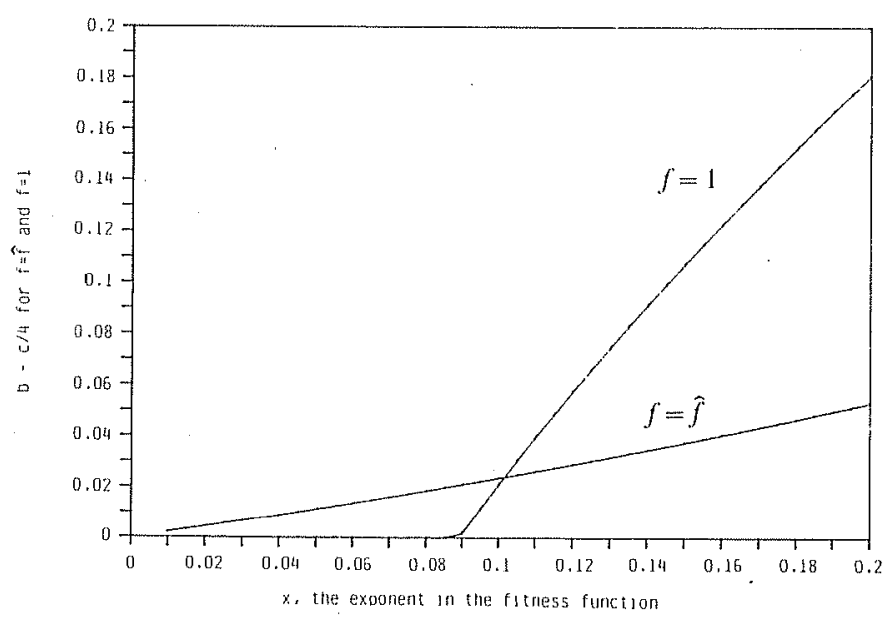

Figure 4 . The value of $b-c / 4$ at $\hat{f}$, the optimum fraction, as a function of $x$ for two-seeded fruits. The value of $b-c / 4$ when $f=1$ (where all the resource is usurped by the dominant seed), also shown in the diagram, is below the former for $x<0.1017$ and above it for higher values of $x$.

This can be better understood from figure 3, which shows the variation of $b-c / 4$ as a function of $f$, the fraction usurped by the dominant seed, for $x=0.095$. Clearly, $f=0.5376$ is the ESS. On the other hand, for $x=0.12$ (figure 3), even though $f=0.5482$ corresponds to a maximum, the value of $b-c / 4$ at $f=1$ is higher than that, indicating $f=1$ to be the ESS. As seen from figure 4 , the value of $b-c / 4$ at $\hat{f}$ is above that corresponding to $f=1$ for values of $x<0.1017$, and is below it for higher values of $x$. In other words, for $x>0 \cdot 1017$, the ESS corresponds to the entire resource being acquired by the selfish seed, thereby leading to brood reduction. 


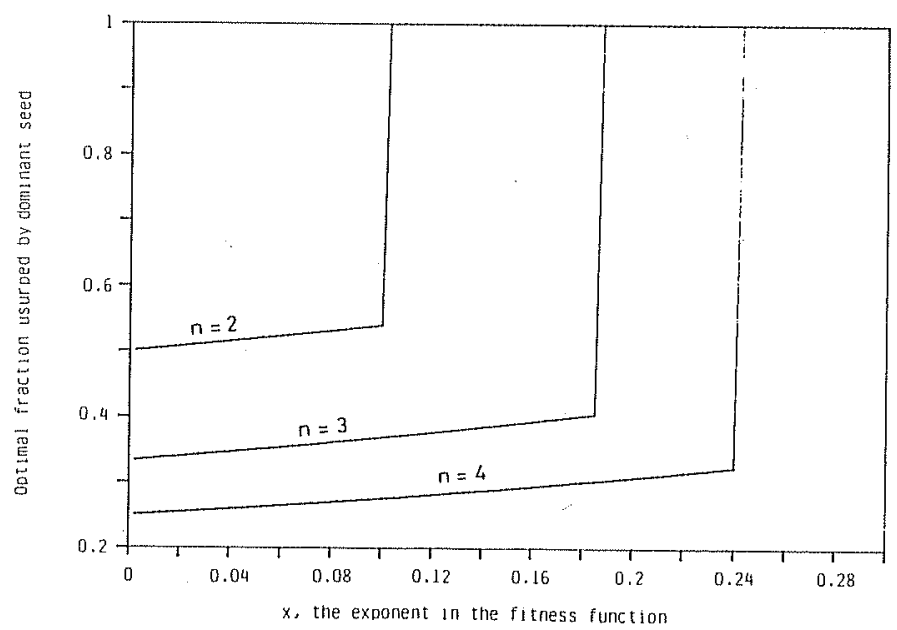

Figure 5. Variation of the optimal fraction $(f=\hat{f}$ or $f=1)$ usurped by the dominant seed at ESS as a function of $x$ for two-seeded, three-seeded, and four-seeded fruits.

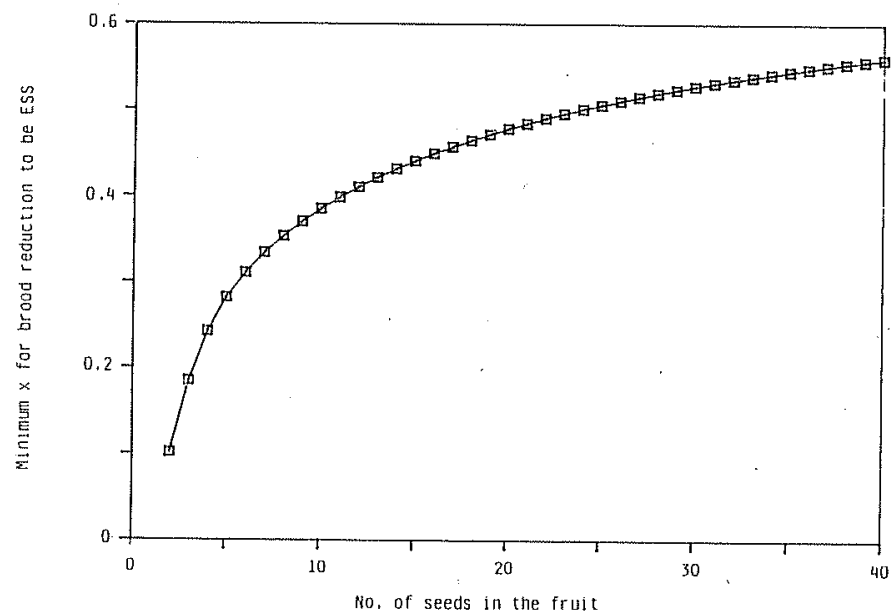

Figure 6. The threshold value of $x$ beyond which brood reduction is the ESS is shown as a function of $n$, the number of seeds in a fruit.

For a fruit with $n$ seeds, the uninvadable strategy (ESS) corresponds to the resource allocation which maximizes $b-(n-1) c / 4$. The optimal allocation in the selfish seed in this situation is seen to be (see appendix, A3)

$$
\widehat{f}=\frac{1}{n}+\frac{(n-1) x\left(1-4^{1 /(x-1)}\right)}{n\left(1+(n-1) 4^{1 /(x-1)}\right)}
$$

The variation of $\hat{f}$ with $x$ for different values of $n$ is shown in figure 5 for $n=2,3$ and 4 . The optimal fraction acquired by the dominant seed for a given value of $x$ is seen to decrease as the number of seeds in a fruit increases, suggesting that the intensity of the conflict among seeds decreases as the number of seeds in a fruit increases. 
The minimum value of $x$ necessary for brood reduction to be the ESS is shown in figure 6 as a function of $n$, the number of seeds in a fruit. The threshold corresponding to a two-seed situation is the lowest, and increases with increasing $n$. This further emphasizes the decrease in the intensity of the seed-seed conflict with increasing number of seeds in a fruit.

\subsection{Predictions of the model and supportive data}

The above analysis indicates that sibling rivalry would lead to brood reduction in two-seeded fruits for a broad range of values of $x$, the exponent in the fitness function (i.e. the efficiency of conversion of resources into fitness). On the one hand, there can be no sibling rivalry in one-seeded fruits. On the other hand, the intensity of sibling rivalry is seen to decrease with an increase in the brood size. In fact, since the inclusive fitness for a selfish allele in an $n$-seeded fruit is $b-(n-1) c / 4$, the weightage given to the cost increases rapidly with $n$, thereby decreasing the propensity for brood reduction. Taken together, these results indicate that sibling rivalry would be most intense in two-seeded fruits. Since under such a scenario, loss of one seed is virtually certain, it would not be in the interest of the parent to produce two-ovuled flowers; either only one or very many would be favourable. The model thus predicts that species with two-ovuled flowers would be rather rare.

An examination of the frequency distribution of ovules per flower and seeds per fruit (table 1; Hegde, Uma Shaanker and Ganeshaiah, personal communication) in over 800 species does reveal the predicted pattern. The frequency of two-ovuled flowers (and two-seeded fruits) is indeed lower than that of one-ovuled or many-ovuled flowers (and one-seeded or many-seeded fruits). Given the complexity of myriad factors that affect ovule and seed number (dispersal mode, habit, packing cost, parent-offspring conflict, etc.: Wiens 1984; Nakamura. 1986; Wiens et al. 1987; Ganeshaiah and Uma Shaanker 1988; Uma Shaanker and Ganeshaiah 1988; Uma Shaanker et al. 1990), and the severe simplifying assumption made in the model, the

Table 1. Frequency distribution of ovules per flower and seeds per fruit*.

\begin{tabular}{lrrrr}
\hline $\begin{array}{l}\text { Size } \\
\text { class }\end{array}$ & $\begin{array}{c}\text { Number of species } \\
\text { with corresponding } \\
\text { number of ovules } \\
\text { per flower }\end{array}$ & \multicolumn{2}{c}{$\begin{array}{c}\text { Number of species } \\
\text { with corresponding } \\
\text { number of seeds } \\
\text { per fruit }\end{array}$} \\
\hline 1 & 60 & $(7.43)$ & 177 & $(21 \cdot 96)$ \\
$1-2$ & 147 & $(18.22)$ & 163 & $(20 \cdot 22)$ \\
2 & 64 & $(7.93)$ & 5 & $(0 \cdot 62)$ \\
$3-5$ & 147 & $(18.22)$ & 93 & $(11.54)$ \\
$>5$ & 389 & $(48.20)$ & 368 & $(45 \cdot 66)$ \\
\hline
\end{tabular}

Numbers in parentheses are percentages.

* Data kindly supplied by Drs S. G. Hegde, R. Uma Shaanker and K. N. Ganeshaiah. 
agreement with the data cannot be said to really validate the model, though it is gratifyingly supportive of it.

\subsection{Concluding remarks}

One of the consequences of brood reduction is the loss of fitness for the parent. The parent-offspring conflict in such a situation is not over the total quantum of investment (since it is assumed to be constant) but over its differential allocation between sibs. A possible counter-strategy of the parent against brood reduction is polyembryony (Ganeshaiah et al. 1991), whereby the parent produces an extra asexual embryo within the seed. While Ganeshaiah et al. (1991) provided arguments as well as some data in support of this assertion, an explicit population genetic model to demonstrate the same was lacking. These investigations are in progress.

The interplay between parent-offspring conflict and sibling rivalry within a fruit is particularly intricate because of the complex genetic structure (Law and Cannings 1984) of the tissues involved, as perceptively pointed out by Haig and Westoby (1988). The situation could become more interesting if the phenomenon of genomic imprinting (Haig and Westoby 1991) also has a role to play in this conflict. Rather careful genetic modelling may be needed to unravel the relative importance of these possible factors.

Remarkably, however, despite the numerous simplifications, the family level selection models pioneered by Haldane continue to be useful in providing insights into the evolutionary forces governing the patterns of brood size in plants.

\section{Acknowledgement}

It is a pleasure to thank Prof. H. Sharat Chandra for the invitation to participate in the Haldane Centenary Symposium, which provided the necessary stimulus to carry out the work described above. The ideas developed here grew out of discussions with Dr R. Uma Shaanker and Dr K. N. Ganeshaiah, University of Agricultural Sciences, Bangalore, who, along with Dr S. G. Hegde, also kindly made available the data on frequency distribution of ovules and seeds. Their comments were helpful in revising the manuscript to make it more comprehensible and also more accurate. I also acknowledge with thanks A. V. Nagarathnamma for her ability, speed and patience in converting an almost illegible set of notes into readable manuscript. Partial financial support from the Ministry of Environment and Forests, Government of India, is gratefully acknowledged.

\section{Mathematical appendix}

Using the same approach as described in section 2 , the conditions on the relative values of benefit and cost for the spread of a selfish allele, for the ESS, and for the threshold for brood reduction are derived here for the general case of $n$ seeds per fruit. As before, it is assumed that in a fruit containing one or more selfish seeds, one of the selfish seeds will acquire a bigger share, and will have a fitness of $1+b$, while each of the rest of $n-1$ seeds have an equal share of the remaining resource, and 
each a fitness $1-c$. Alternative ways of sharing resource may easily be imagined; these would form the subject of a separate investigation.

\section{A1. Spread of the selfish allele}

Let $y(\simeq 0)$ be the frequency of the NS heterozygote, which implies the frequency of pollen to be $y / 2$. As before, analysis is carried out to first order only.

Contributions to the frequency of $S$ in the next generation via pollen will be through the fertilization of $(\mathrm{N}, \mathrm{N}, \ldots, \mathrm{N})$ flowers by a single $\mathrm{S}$ pollen (and $n-1$ pollen of type $\mathrm{N})$. The frequency of such flowers will be

$$
(y / 2) n(1-y)=n y / 2,
$$

and the fitness of the single NS seed is $1+b$.

The contributions via ovules will be through the $n$ kinds of flowers, containing $1,2, \ldots, n$ ovules of haplotype $\mathrm{S}$. Since all these will be fertilized by $\mathrm{N}$-type pollen, there would be $n$ kinds of fruits, containing $1,2, \ldots, n$ seeds with genotype NS. The frequency $p(k)$ of fruits with $k$ seeds with genotype NS is given, from binomial distribution, by

$$
p(k)=\left(\frac{n !}{k !(n-k) !}\right)\left(\frac{1}{2^{n}}\right) y .
$$

The fitness of an NS seed in such a fruit is given by

$$
f(k)=1+b / k-c(1-1 / k) \text {. }
$$

Hence the frequency of the NS genotype in the next generation is given by

$$
\begin{aligned}
y^{\prime} & =y\left[\frac{n}{2}(1+b)+\sum_{k=1}^{n} p(k) f(k) k\right] / n \\
& =y\left[\frac{n}{2}(1+b)+\frac{1}{2^{n}} \sum_{k=1}^{n} \frac{n !}{k !(n-k) !}(k+b-c(k-1))\right] / n \\
& =y\left[\frac{n}{2}(1+b)+\frac{(b+c)}{2^{n}} \sum_{k=1}^{n} \frac{n !}{k !(n-k) !}+\frac{(1-c)}{2^{n}} \sum_{k=1}^{n} \frac{n !}{k !(n-k) !} k\right] / n .
\end{aligned}
$$

The first summation equals $2^{n}-1$, while the second equals $n\left(2^{n-1}\right)$. Substituting and simplifying,

$$
y^{\prime}=y\left[n+(b+c)\left(1-\frac{1}{2^{n}}\right)+(b-c) \frac{n}{2}\right] / n,
$$

and thus $y^{\prime}$ will be greater than $y$ if

$$
(b+c)\left(1-\frac{1}{2^{n}}\right)+(b-c) \frac{n}{2}>0 .
$$

Further simplification shows the condition for the spread of the selfish allele to be

$$
b>c\left(\frac{2^{n-1}(n-2)+1}{2^{n-1}(n+2)-1}\right)
$$




\section{A2. Evolutionarily stable strategy}

As in section 2 , let $z(\simeq 0)$ be the frequency of $\mathrm{SS}^{\prime}$ genotypes, which implies the frequency of $\mathrm{S}^{\prime}$ pollen to be $z / 2$.

Arguing along the lines similar to the ones in section A1, the contributions to the next generation of $S^{\prime}$ via pollen will come from fruits with one $\mathrm{SS}^{\prime}$ seed (and $n-1$ seeds of SS genotype), whose fitness would be $1+b^{\prime} / n-c(1-1 / n)$.

This is because, the probability that the SS' seed will be the "winner" is $1 / n$, and the corresponding fitness is $1+b^{\prime}$. The probability that the winner is SS is $(n-1) / n$, and the fitness to $\mathrm{SS}^{\prime}$ in such a situation is $1-c$.

For the contribution to the next generation via ovules, the frequency of flowers containing $k$ pollens of type $\mathrm{S}^{\prime}$ is given by equation (ii) (except for $z$ in place of $y$ ). However, the fitness of an $\mathrm{SS}^{\prime}$ seed from such a fruit is

$$
f(k)=1+\frac{b^{\prime}}{n}-c^{\prime}\left(\frac{k-1}{n}\right)-c\left(\frac{n-k}{n}\right) .
$$

The net fitness of the $\mathrm{SS}^{\prime}$ genotype is given by

$$
\begin{aligned}
& {\left[n \frac{z}{2}\left\{1+\frac{b^{\prime}}{n}-c\left(1-\frac{1}{n}\right)\right\}+z \sum_{k=1}^{n} p(k) f(k) k\right] / z} \\
& \quad=n+b^{\prime}-\frac{3}{4}(n-1) c-\frac{(n-1) c^{\prime}}{4} .
\end{aligned}
$$

This has to be greater than the fitness of the SS genotype which is $n+b-(n-1) c$. Hence, fitness of $\mathrm{SS}^{\prime}>$ fitness of SS if

$$
b^{\prime}-(n-1) c^{\prime} / 4>b-(n-1) c / 4
$$

Thus, the strategy which maximizes $b-(n-1) c / 4$ would be able to invade any other strategy, and will be uninvadable by any other strategy, and hence will be the ESS.

\section{A3. Threshold for brood reduction}

The net resource invested by the parent in the fruit with $n$ seeds is (section 2.4) equal to $n /(1-x)$. If the dominant seed usurps a fraction $f>1 / n$, its fitness is

$$
F^{\prime}=\left(\frac{f n}{1-x}-1\right)^{x}
$$

and the fitness of each of the rest of the seeds is

$$
F^{\prime \prime}=\left(\frac{(1-f) n}{(n-1)(1-x)}-1\right)^{x}
$$

These can be used to derive $\hat{f}$, the optimal allocation, in the same manner as in 
section 2.4 , the expression for $\hat{f}$ being

$$
\hat{f}=\frac{1}{n}+\frac{(n-1) x\left(1-4^{1 /(x-1)}\right)}{n\left(1+(n-1) 4^{1 /(x-1)}\right)} .
$$

Let $b$ and $c$ be the corresponding benefits and costs.

As long as $f<(n-1) / n$, each of the seeds other than the dominant obtains at least one unit of resource, and is thus viable. For $f>(n-1) / n$, only one seed survives, and brood reduction takes place.

When the entire resource is usurped by the dominant seed $(f=1)$, its fitness is

$$
F^{*}=\left(\frac{n}{1-x}-1\right)^{x} \text {. }
$$

Let $b^{*}$ be the benefit, while the cost $c$ is unity.

If $b^{*}-(n-1) / 4$ is greater than $b-(n-1) c / 4$, then $f=1$ is the ESS, and brood reduction takes place; only the dominant seed survives and the rest are aborted.

These criteria may be used to obtain the threshold value of $x$ for brood reduction to occur in $n$-seeded fruits.

\section{References}

Ganeshaiah K. N. and Uma Shaanker R. 1988 Regulation of seed number and female incitation of mate competition by a $\mathrm{pH}$-dependent proteinaceous inhibitor of pollen grain germination in Leucaena leucocephala. Oecologia 75: 110-113

Ganeshaiah K. N., Uma Shanker R. and Joshi N. V. 1991 Evolution of polyembryony: Consequences to the fitness of mother and offspring. J. Genet. 70: 103-127

Haig D. and Westoby M. 1988 Inclusive fitness, seed resources, and maternal care. In Plant reproductive ecology (eds) Jon Lovett Doust and Lesley Lovett Doust (New York: Oxford University Press) pp. 60-79

Haig D. and Westoby M. 1991 Genomic imprinting in endosperm: its effect on seed development in crosses between species, and between different ploidies of the same species, and its implications for the evolution of apomixis. Phil. Trans. R. Soc. London B333: 1-13

Haldane J. B. S. 1924 A mathematical theory of natural and artificial selection. Part I. Trans. Cambridge Philos. Soc. 23: 19-41

Hamilton W. D. 1964 The genetical evolution of social behaviour, I. J. Theor. Biol. 7: 1-16

Hamilton W. D. 1964 The genetical evolution of social behaviour, II. J. Theor. Biol. 7: 17-52

Kress W. J. 1981 Sibling competition and evolution of pollen unit, ovule number and pollen vector in angiosperms. Syst. Bot. 6: 101-112

Law R. and Cannings C. 1984 Genetic analysis of conflicts arising during development of seeds in the Angiospermophyta, Proc. R. Soc. London B221: 53-70

Lee T. D. 1988 Patterns of fruit and seed production. In Plant reproductive ecology (eds) Jon Lovett Doust and Lesley Lovett Doust (New York: Oxford University Press) pp. 179-202

Lovett Doust J. and Lovett Doust L. 1988 Sociobiology of plants: an emerging synthesis. In Plant reproductive ecology (eds) Jon Lovett Doust and Lesley Lovett Doust (New York: Oxford University Press) pp. 5-29

Macnair M. R. and Parker G. A. 1979 Models of parent--offspring conflict. III. Intra-brood conflict. Anim. Behav. 27: 1202-1209

Nakamura R. R. 1980 Plant kin selection. Evol. Theory 5: 113-117

Nakamura R. R. 1986 Maternal investment and fruit abortion in Phaseolus vulgaris. Am. J. Bot. 73: 1049-1057

O'Connor R. J. 1978 Brood reduction in birds: selection for fratricide, infanticide and suicide. Anim. Behav, 26: 79-96

Queller D. C. 1983 Kin selection and conflict in seed maturation. J. Theor. Biol. 100: 153-172

Smith C. C. and Fretwell S. D. 1974 The optimal balance between size and number of offspring. Am. Nat. 108: 499-506 
Stinson C. H. 1979 On the selective advantage of fratricide in raptors. Evolution 33: 1219-1225

Sutter J. 1969 Haldane and demographic genetics. In Haldane and modern biology (ed) K. R. Dronamraju (Baltimore: The Johns Hopkins Press) pp. 73-77

Uma Shaanker R. and Ganeshaiah K. N. 1988 Bimodal distribution of seeds per pod in Caesalpinia pulcherrima: parent-offspring conflict? Evol. Trends Plants 2: 91-98

Uma Shaanker R., Ganeshaiah K. N. and Bawa K. S. 1988 Parent-offspring conflict, sibling rivalry, and brood reduction in plants. Annu. Rev. Ecol. Syst. 14: 177-205

Uma Shaanker R., Ganeshaiah, K. N. and Radhamani T. R. 1990 Associations among the modes of pollination and seed dispersal--ecological factors and phylogenetic constraints. Evol. Trends Plants 4: 107-111

Westoby M. and Rice B. 1982 Evolution of seed plants and inclusive fitness of plant tissues. Evolution 36: $713-724$

Wiens D. 1984 Ovule survivorship, brood size, life history, breeding systems and reproduction success in plants. Oecologia 64: 47-53

Wiens D., Calvin C. L., Wilson C. A., Davern C. I., Frank D. and Seavey S. R. 1987 Reproductive success, spontaneous embryo abortion and genetic load in flowering plants. Oecologia 71: 501-509 